\title{
Anxiety in Late Life: An Update on Pathomechanisms
}

\author{
Sabine Hellwig ${ }^{a}$ Katharina Domschke ${ }^{a, b}$ \\ ${ }^{a}$ Department of Psychiatry and Psychotherapy, Medical Center - University of Freiburg, Faculty of Medicine, \\ University of Freiburg, Freiburg, Germany; ${ }^{\mathrm{b}}$ Center for Basics in Neuromodulation, Faculty of Medicine, University of \\ Freiburg, Freiburg, Germany
}

\section{Keywords}

Anxiety disorders - Late life · Elderly · Older adults ·

Comorbidity · Neuroimaging · Neuroendocrinology ·

Neuropsychology

\section{Abstract}

Anxiety disorders are common, yet clinically underrecognized in late life, with estimated prevalence rates ranging from 1.2 to $15 \%$. They are highly comorbid with depression, sleep disorders, and substance use disorders, may accelerate cognitive decline, and potentially catalyze morbidity and mortality risk in the elderly. Thus, a more detailed knowledge about the underlying mechanisms of late-life anxiety disorders is urgently warranted. Age-related genetic, neuroimaging, neuroendocrine, and neuropsychological markers as well as late-life specific psychosocial aspects, particularly loss and isolation, have been identified as prominent pathogenetically relevant and thus potentially targetable factors. Personalized treatments based on individual biological and biographic markers, innovative therapeutic approaches, and preventive strategies have great potential to alleviate the high individual and societal burden of late-life anxiety disorders.

(c) 2019 S. Karger AG, Basel

\section{Introduction}

When contemplating the definition and measurement of late-life anxiety, it is important to consider "normal" anxiety revolving around social isolation, health issues, and mortality as an adaptive process inherent to aging and coping with aging. Anxiety is furthermore a prominent symptom in patients diagnosed with any mental, neurodegenerative, or somatic disorder, leading to the inclusion of anxiety as a cross-cutting symptom measure in the current version of the Diagnostic and Statistical Manual of Mental Disorders (DSM-5). Exceeding "normal" and symptomatic anxiety, anxiety disorders as categorical nosological entities represent a major clinical challenge in late life, with estimated prevalence rates ranging from 1.2 to $15 \%$. Among anxiety disorders according to DSM-5 criteria, in late life agoraphobia has been found to occur most frequently $(4.9 \%)$, followed by panic disorder (3.8\%), generalized anxiety disorder (GAD) (3.1\%), specific phobias (2.9\%), and social phobia (1.3\%). Furthermore, late-life anxiety disorders are significantly associated with comorbid major depression. First-line treatment of anxiety disorders in old age comprises pharmacological treatment with selective serotonin reup-

\section{KARGER}

(C) 2019 S. Karger AG, Basel

E-Mail karger@karger.com

www.karger.com/ger
Katharina Domschke, $\mathrm{MD}, \mathrm{PhD}$

Department of Psychiatry and Psychotherapy

Medical Center - University of Freiburg

Hauptstrasse 5, DE-79104 Freiburg (Germany)

E-Mail katharina.domschke@ uniklinik-freiburg.de 
take inhibitors, serotonin and norepinephrine reuptake inhibitors, and - in GAD - pregabalin, as well as psychotherapeutic interventions, mostly cognitive behavioral therapy (CBT) [reviewed in 1].

In clinical practice, late-life anxiety disorders constitute largely undetected and untreated disorders, although it is increasingly known that their disease burden and their relevance for psychiatric or somatic comorbidity and mortality are considerable. For instance, late-life GAD has been shown to be associated with a significantly increased risk of sleep maintenance insomnia, initial insomnia, and early morning awakening [2]. Old-age female patients with panic disorder display elevated odds of developing dependence on benzodiazepines [3]. Furthermore, prospective studies point to a negative [reviewed in 4] and potentially also prognostic impact of anxiety symptoms on cognitive function in late life, with recent neuroimaging studies suggesting that anxiety symptoms could be a prodromal marker or even an independent risk factor for Alzheimer's diseases as well as vascular dementia $[5,6]$. Resting activity of the amygdala as a neural correlate of emotional distress as conferred by anxiety has been associated with an increased number of cardiovascular disease events potentially via heightened bone marrow activity and arterial inflammation [7], and elevated trait anxiety in late life seems to increase the risk of coronary artery calcification [8]. Finally, while overall evidence of late-life anxiety confering increased mortality is inconclusive or restricted to men $[9,10]$, in a recent study higher levels of psychological distress including anxiety and depression symptoms were shown to predict site-specific cancer mortality particularly pertaining to carcinoma of the colorectum, prostate, pancreas, and oesophagus as well as leukemia [11].

Against this background, the present review on latelife anxiety disorders is intended to provide a focused update on mechanisms underlying experimental, clinical, and behavioral aspects of anxiety explicitly in old age, since aging is associated with individual age-associated changes in anxiety-related biological risk factors (e.g., hormones and neuronal function) and exposure to agespecific psychosocial influences (e.g., living alone, physical illness, and disability or cognitive decline) potentially informing age-specific diagnostic and therapeutic processes. Along these lines, the necessity to improve diagnostic methods specific to late-life anxiety and the promising role of personalized and innovative treatments as well as preventive strategies regarding late-life anxiety and thereby potentially also its sequential comorbid conditions will be outlined.

\section{Pathomechanisms of Anxiety in Late Life}

The pathogenesis of anxiety disorders in general is multifactorial, involving complex interactions between biological factors, environmental influences, and psychological mechanisms. The present review focuses on recent advances in uncovering biological as well as some psychological and environmental mechanisms of anxiety specifically in late life. A model summarizing and schematically illustrating the mechanisms underlying late-life anxiety as reviewed in the following paragraphs is given in Figure 1.

\section{Genetics}

Family and twin studies indicate a substantial contribution of genetic factors in the pathogenesis of anxiety disorders (heritability estimates derived from twin studies range from $32 \%$ for GAD to $67 \%$ for agoraphobia), with the remaining variance being attributed to environmental influences [for review see 12]. Of particular relevance to late-life anxiety, a twin study based on data from 1,482 Swedish participants aged $\geq 50$ years investigating the trajectory of anxiety symptoms over the course of 11 years revealed the influence of genetic variance to increase at an accelerated pace from approximately age 60 years onward [13], underscoring the high relevance of biological components in the pathogenesis of late-life anxiety. On a candidate gene level, APOE $\varepsilon 4$ allele carriage among amyloid- $\beta(A \beta)$-positive older adults was associated with an increased severity of anxiety symptoms as assessed using the Hospital Depression and Anxiety Scale (HADS), with this effect being particularly prominent in the female subgroup [14]. This is in line with a Pittsburgh compound $\mathrm{B}(\mathrm{PiB})$ positron emission tomography (PET) study measuring the cortical $A \beta$ load in 118 cognitively normal elderly volunteers (aged $65-90$ years), which revealed an association between anxiety measures (HADS) and the subcortical A $\beta$ burden. Higher PiB PET measures in the striatum, amygdala, and thalamus, but not in the cortex, were associated with greater anxiety when adjusting for demographics, cognition, and depression. The highest anxiety levels were found in APOE $\varepsilon 4$ allele carriers with subcortical amyloidosis [6]. These genetic findings in synopsis with results from imaging studies underline a potentially shared pathogenetic pathway for anxiety and cognitive impairment as preliminarily suggested by clinical (see Introduction) and neuropsychological studies (see below).

The only available pharmacogenetic study on late-life anxiety investigating 150 GAD participants aged $\geq 60$ 


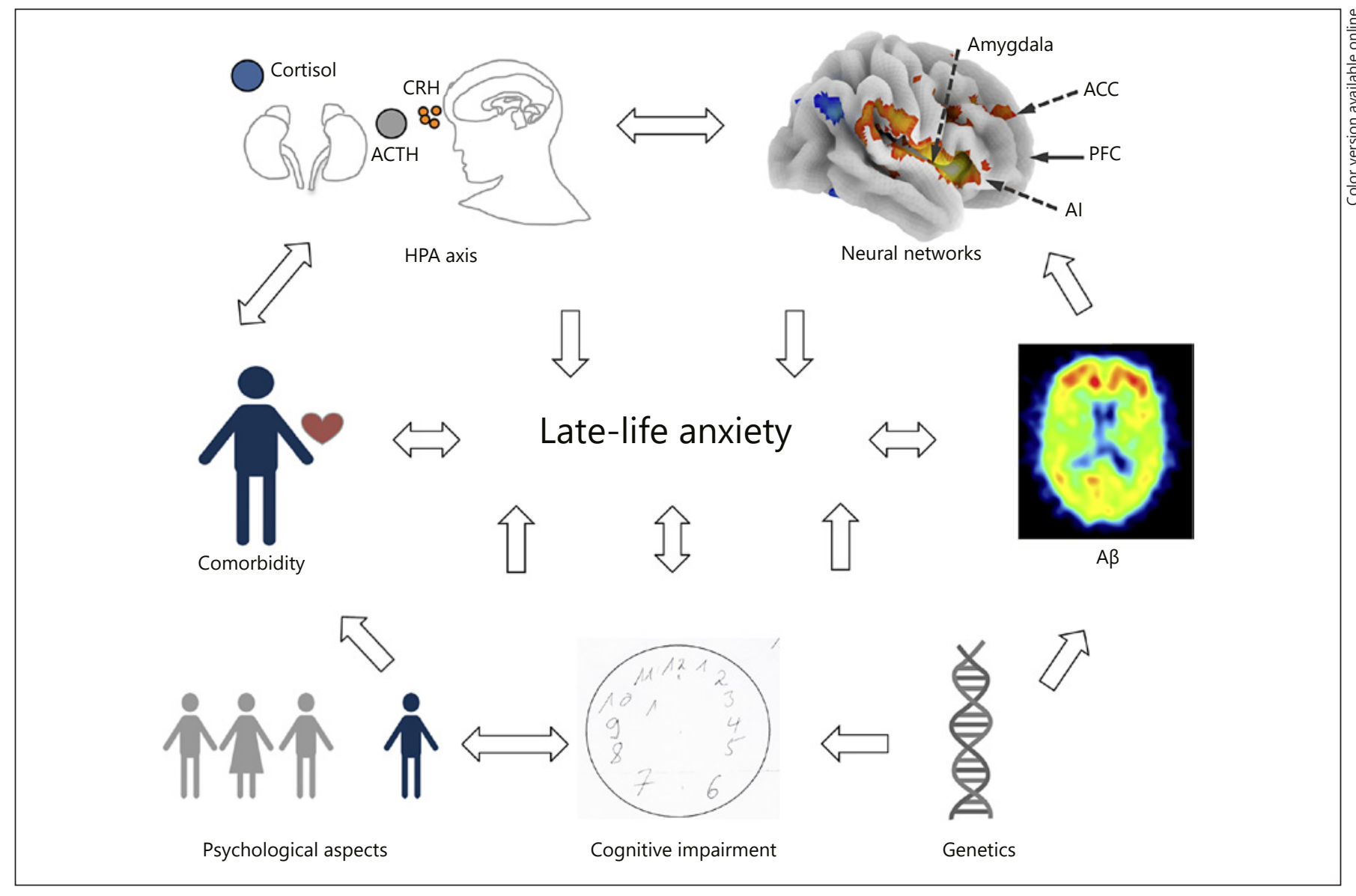

Fig. 1. Mechanistic model of anxiety in late life. ACTH, adrenocorticotropic hormone; $\mathrm{CRH}$, corticotropin-releasing hormone. For details, please refer to "Pathomechanisms of Anxiety in Late Life".

years enrolled into a 12-week randomized controlled trial of escitalopram versus placebo revealed that the serotonin transporter gene HTTLPR/rs25531 genotype modulated the therapeutic efficacy of escitalopram, with no response in low-activity $\mathrm{S}_{\mathrm{A}}, \mathrm{S}_{\mathrm{G}}$, and $\mathrm{L}_{\mathrm{G}}$ genotype carriers compared to moderate efficacy in the group carrying the $\mathrm{L}_{\mathrm{A}}$ genotype [15]. In the Vienna Transdanube Aging (VITA) study analyzing 544 probands aged 75 years, however, no association of the 5-HTTLPR polymorphism with anxiety as measured by the Spielberger State-Trait Anxiety Inventory (STAI-S/T) could be discerned [16]. These inconsistent findings could be explained by the fact that the influence of 5-HTT gene variation on anxiety has been shown to be strongly modifiable by environmental factors. So-called gene-environment $(\mathrm{G} \times \mathrm{E})$ approaches have revealed an interaction between the less active alleles of the 5-HTTLPR variant and - as particularly relevant to old age - separation life events to increase the risk for panic disorder, while beneficial person characteristics such as coping strategies might buffer vulnerability to anxiety as conferred by the interaction of childhood adversity and the 5-HTTLPR genotype [17]. These approaches have not been applied to anxiety specifically in late life yet; however, taking into account accumulated life events and coping strategies might be of particular relevance in that stage of life. Furthermore, to the best of our knowledge, no genome-wide association studies in latelife anxiety disorders are available, and epigenetic studies are entirely lacking. Thus, a comprehensive evaluation of the (epi)genetic underpinnings of late-life anxiety is not possible given the current state of research.

\section{Neural Networks}

Neuroimaging using magnetic resonance imaging (MRI) allows visualization of structural changes. In latelife GAD participants, a lower cortical thickness in the orbitofrontal cortex (OFC), the inferior frontal gyrus, and the anterior cingulate cortex (ACC) was observed using 
structural MRI, with higher worry severity correlating with gray matter changes in the OFC, the ACC, and the putamen [18].

Task-related brain functions that are not necessarily related to brain structure can be visualized by blood oxygen level-dependent (BOLD) functional MRI (fMRI). In fMRI studies investigating late-life anxiety, particular attention has been paid to exploring neural correlates of specifically worry-related emotion reactivity and regulation. During phases of worry modulation, time series analysis of nonanxious controls revealed that amygdala and subgenual ACC activate in reverse synchronicity, whereas elderly GAD participants exhibited the same activation direction during worry induction [19]. Using an fMRI method quantitatively measuring cerebral blood flow $(\mathrm{CBF})$ as a surrogate of regional neuronal activity (arterial spin labeling), Andreescu et al. [20] demonstrated that late-life GAD subjects failed to activate prefrontal cortical (PFC) regions involved in the downregulation of negative emotions. Furthermore, GAD participants displayed higher CBF levels in multiple regions during worry induction (visual and parietal cortex, middle and superior frontal) and lower CBF levels during reappraisal (supplemental motor area, middle cingulate gyrus, and insula and putamen) when compared to nonanxious controls. Repetitive blocks of worry induction and reappraisal induced no CBF changes, except in the thalamus [21]. Furthermore, late-life GAD participants displayed greater functional connectivity (FC) during worry induction between the left anterior insula (AI) and the right OFC and between the bed nucleus of the stria terminalis and the subgenual ACC [22]. During worry reappraisal, the nonanxious participants displayed greater FC between the left dorsolateral PFC and the medial PFC, as well as between the left AI and the medial PFC, while late-life GAD subjects showed greater FC between the paraventricular nucleus and the amygdala. Following successful pharmacological treatment with citalopram, greater FC in the prefrontal nodes of the executive control network during reappraisal of worry was observed [22].

Parallel to task-related fMRI, resting state fMRI captures spontaneous fluctuations of the BOLD signal during resting state conditions. A resting-state fMRI study in late-life GAD highlighted that disease duration as well as the presence and the severity of worries exacerbate agerelated effects on FC in the default mode network [23].

Besides altered worry-related emotion reactivity and regulation, the erroneous or biased interpretation of stimuli as dangerous or threatening to an individual's physical or psychological well-being ("attention bias") has been proposed as a core feature of anxiety disorders [24]. Accordingly, an fMRI study investigating neural correlates of attention bias in $16 \mathrm{GAD}$ patients (mean age: 66 years) and 12 nonanxious controls discerned that, during presentation of negative words (in comparison with neutral), the control group showed PFC activation coupled with deactivation of task-irrelevant emotional processing regions such as the amygdala and the hippocampus. In contrast, GAD participants showed PFC decreases in response to negative words and no differences in amygdalar activity across word types. A significant positive correlation between attentional bias and amygdala activation was also present, but this relationship was mediated by PFC activity [25].

In summary, neuroimaging studies consistently implicate a dysfunctional interaction between the anxiety-inducing extended limbic (amygdala, bed nucleus of the stria terminalis, and insula) and anxiety-attenuating cortical (PFC, OFC, and ACC) systems as a potential neural correlate of failure to regulate worry in late-life anxiety disorders.

\section{Neuroendocrinology and Hormones}

Age-related alterations in hypothalamic-pituitary-adrenal (HPA) axis function may increase the susceptibility to HPA dysregulation as a neurobiological correlate of stress resilience and thus a potential mediator of mood and anxiety disorders. However, the available data on the role of HPA axis dysfunction in late-life anxiety disorders is sparse. Elevated basal salivary cortisol levels, with higher peak cortisol levels and larger areas under the curve, were associated with the diagnosis of late-life GAD in 71 GAD subjects compared to 40 nonanxious controls [26]. Moreover, the severity of GAD as measured by the GAD severity scale (GADSS) and the Penn State Worry Questionnaire (PSWQ) was positively correlated with cortisol levels [26]. In line with these findings, a randomized controlled trial in 60 adults diagnosed with late-life GAD revealed a decrease in salivary cortisol during a 12-week treatment with escitalopram versus placebo. Treatmentinduced cortisol changes were significantly associated with changes in immediate and delayed memory, but no association with executive tasks (measures of working memory and set shifting) was discerned. Analyses suggested that memory improvement was seen along with cortisol reduction among patients receiving escitalopram but not among patients receiving placebo [27]. Consistently, in a 16-week randomized study with either 16 sessions of CBT in addition to escitalopram or escitalopram 
monotherapy, CBT augmentation of selective serotonin reuptake inhibitor treatment reduced the peak cortisol levels in 42 participants with late-life GAD [28]. Taken together, the burgeoning evidence for a role of HPA axis dysregulation and its implications for treatment in latelife anxiety disorders merits further investigation.

On a hormonal level, a potential role of estradiol in anxiety has been suggested, since it modulates fear learning and fear extinction [29]. The Study of Women's Health Across the Nation (SWAN) suggests a crucial role of changes in hormonal levels across the menopausal transition in mediating increased anxiety symptoms in women [30]. Accordingly, panic attacks have been shown to arise or worsen with menopause, in correlation with a greater severity of the climacteric syndrome [31]. Consistently, in the Kronos Early Estrogen Prevention Study (KEEPS), recently postmenopausal women randomized to oral conjugated equine estrogens showed improvement of depressive and anxiety symptoms compared to placebo over a 4-year treatment period [32]. However, a prospective study including 838 postmenopausal women aged $\geq 65$ years indicated no significant association between hormone therapy and anxiety disorders at baseline or after the 4-year follow-up period, irrespectively of the type of treatment (oral as well as transdermal application of estradiol alone, estradiol combined with progesterone, or synthetic progestin) [33]. Thus, the role of hormonal changes after menopause and the potential of hormone replacement therapy in late-life anxiety disorders in females remain to be elucidated in more depth [for review see 34].

\section{Neuropsychology}

Cluster A and B personality disorders such as schizotypal, borderline, and narcissistic personality disorders were identified as predictors of incident panic disorder, social phobia, and GAD, respectively, in a nationally representative sample of 8,012 community-dwelling adults aged $\geq 60$ years interviewed twice over a period of 3 years [35].

Furthermore, anxiety symptoms are more often present in older adults with mild cognitive impairment or dementia than in older adults with normal cognition [36], and cognitive impairment precipitated phobic anxiety symptoms in 64 community-dwelling older adults aged $\geq 50$ years [37]. A recent risk-modelling study indicated that age-associated changes in cognitive performance contributed to the development of anxiety symptoms as measured by the Hamilton Anxiety Rating Scale (HARS) scores in adults aged $\geq 65$ years with specific negative af- fect trait characteristics as assessed by the Negative Affect Scale score of the Positive and Negative Affect Schedule (PANAS-N) [38]. Moreover, cognitive impairment has been proposed as a negative prognostic factor for late-life anxiety. In a pilot study (in 32 adults aged $\geq 60$ years diagnosed with GAD) participants with persistent executive dysfunction did not respond to CBT, while GAD participants without executive deficits had a therapeutic benefit [39]. Establishing a causal relationship between cognitive impairment and anxiety, however, is still a subject of research, as current literature points toward a bidirectional nature (Fig. 1); while cognitive impairment has been proposed as a negative prognostic factor for latelife anxiety, anxiety has reciprocally been linked to a poorer prognosis regarding cognitive function (see Introduction).

Finally, information processing biases have been implicated as both causal and maintenance factors in cognitive models of anxiety (see above) [24]. Accordingly, processing bias was evaluated in a sample of 25 older adults with GAD during a 12-week treatment with escitalopram. Steiner et al. [40] found that a more positive bias score across time predicted a better treatment response. Faster responses to positive words relative to negative words were associated with greater symptomatic improvement over time as reflected by scores on the GADSS.

\section{Psychosocial Factors}

Late-life specific psychosocial aspects, particularly loss and social isolation (i.e., loneliness), have been linked to anxiety. With age, the likelihood of experiencing agerelated losses (e.g., death of a spouse and friends and social disengagement after leaving work or a familiar neighborhood) increases. Age-related losses impede maintenance and acquisition of relationships, leading to a higher prevalence of loneliness. Current estimates indicate an overall prevalence rate of loneliness of up to $29 \%$ among older adults in the USA. Prevalence rates in European countries vary between 20 and $34 \%$ [reviewed in $41]$. In a prospective cohort study comprising 594 primary care patients aged 18-65 years and diagnosed with anxiety or depressive disorders at baseline as ascertained by the Beck Anxiety Inventory (BAI) and the Inventory of Depressive Symptomatology (IDS), loneliness was an independent predictor of poorer symptom outcomes regarding anxiety and depression at the 1-year follow-up [42]. A subsequent analysis of data derived from that cohort revealed that the risk of admission for anxiety disorders was significantly associated with the presence of loneliness [43]. 


\section{Discussion and Outlook}

It is increasingly acknowledged that anxiety disorders are frequent disorders in late life and of paramount prognostic impact as an underrecognized cause of mental and physical disability as well as a potential catalyst of the mortality risk in the elderly, warranting a heightened awareness and improved diagnostic methods among clinicians. Late-life anxiety might be understood as the result of several simultaneously working processes. On one hand, aging is associated with age-specific psychosocial risk factors for anxiety (i.e., living alone, physical illness and disability, or cognitive decline). On the other hand, aging may protect against anxiety by age-specific coping strategies leading to increased resilience and changes in life perspective rendering common threats less anxiety provoking. Individual age-associated anxiety-related biological determinants (i.e., APOE $\varepsilon 4$ allele, hormonal decline, altered HPA axis function, and impaired neuronal function) and selective survivorship of those who are less prone to anxiety (mortality aspect) further modulate the spectrum between risk and resilience. Recent promising studies focusing on genetics, neuroimaging, neuroendocrinology, and neuropsychology as well as age-specific psychosocial aspects as reviewed above (also see Fig. 1) have revealed some of the potential underlying mechanisms of late-life anxiety disorders, which eventually may inform personalized treatment, innovative therapeutic approaches, and prevention of late-life anxiety. Interestingly, paralleling the higher prevalence of anxiety disorders in females than in males, female-specific biological and social factors have been identified to modulate the course of anxiety disorders over the female lifespan. Women are more likely to experience stressors that may precipitate anxiety disorders, exhibit cognitive styles prone to rumination and worry, and show biological predispositions (e.g., anxiety sensitivity and hormonal fluctuations) that contribute to anxiety disorders [reviewed in 34]. However, the present body of evidence for mechanisms specific to late-life anxiety disorders is still only burgeoning and thus has to be considered hypothesis generating, warranting further experimental investigation. The currently necessary next steps and possible clinical applications derived from mechanistic research in the field of late-life anxiety are discussed below.

\section{Improved Diagnostic Methods}

To facilitate the accurate diagnosis of late-life anxiety disorders as well as research into its potentially specific pathomechanisms, an Advisory Committee to the Lifes- pan Disorders Work Group proposed changes to the current DSM-5 criteria [44]. First, recommendations regarding age-specific assessment of avoidance and excessive behavior are given; both are considered essential elements in the conceptualization and diagnosis of anxiety disorders. Second, the demand for guidelines on the evaluation of comorbid anxiety and depression in older adults is highlighted, and an age-sensitive version of the category "mixed anxiety depression" is propagated. Third, suggestions for age-specific measurement of the criteria "impairment," "distress," and "excessive anxiety" are made, and elimination of the "insight" criterion (e.g., specific phobia, social phobia) is suggested. Fourth, assessment of anxiety in the context of medical illness and cognitive impairment/dementia is reinforced. Fifth, an extension of DSM-5 criteria by inclusion of late-life specific variants of anxiety disorders (e.g., fear of falling) is encouraged. Finally, detailed suggestions to improve the sensitivity of measurement instruments are made, and adequate representation of older adults with anxiety disorders in field trials is recommended [44]. Along these lines, 2 age-specific measures achieved encouraging results in the differential diagnostics of late-life anxiety disorders, i.e., the 20-item Geriatric Anxiety Inventory (GAI) modelled after the Geriatric Depression Scale (GDS), using a simple response scale of "agree" or "disagree" [45], and the 30-item Geriatric Anxiety Scale (GAS) assessing somatic, cognitive (with emphasis on worrying), and affective anxiety symptoms rated on a dimensional, 4-point scale of anxiety severity [46]. Psychometric studies underscored excellent internal consistency and strong convergent validity with measures of anxiety and worry for both the GAI [45] and the GAS [46]. Moreover, the GAI has been shown to ascertain anxiety levels in elderly with cognitive impairment and patients in long-term care facilities [47].

\section{Personalized Treatment}

Research into (bio)marker-based prediction of the therapy response, tolerability, and side effects can be helpful in individually tailoring treatment of late-life anxiety, extending available evidence in early and mid-life anxiety disorders [48]. For instance, based on a study by Steiner et al. [40], a more positive processing bias might serve as a marker of the treatment response to escitalopram in old-age GAD. Furthermore, a pharmacogenetic study in late-life anxiety suggest that the 5-HTTLPR/ rs25531 genotype modulates the therapeutic efficacy of escitalopram [15]. These findings might contribute to a better understanding of antianxiety treatment mecha- 
nisms and possibly allow for the identification of response predictors to be utilized in more personalized and thus more effective treatment approaches in late-life anxiety. However, the epistatic effects of 5-HTT gene variation with further polymorphisms in the 5-HTT gene and in other genes related to anxiety pathology or antianxiety treatment (for review see [12]) as well as the dynamic epigenetic variation crucially governing gene function and reflecting the lifetime impact of environmental and somatic factors as particularly relevant in older age [17] warrant investigation in future, statistically sufficiently powered studies, ideally adopting a systems perspective integrating a wealth of biological as well as biographical information.

\section{Innovative Treatment}

On a pharmacological level, taking into account that HPA axis dysfunction with elevated cortisol levels has been linked to late-life anxiety (please refer to Neuroendocrinology and Hormones in Results), a pilot study with the glucocorticoid receptor antagonist mifepristone was conducted in 15 patients aged $\geq 60$ years with an anxiety disorder and cognitive dysfunction. As expected, a 12week treatment with $300 \mathrm{mg}$ mifepristone induced an increase in cortisol levels, which returned to baseline levels after drug cessation. Participants with higher baseline cortisol levels improved in memory, executive function, and worry severity as assessed by a significant reduction in PSWQ-Abbreviated (PSWQ-A) scores, with persistent benefits after discontinuation of the antiglucocorticoid agent. In contrast, slight to no improvement was observed among individuals with low to normal baseline cortisol levels [49].

Among psychotherapeutic approaches targeting anxiety in late-life, the use of telephone-delivered psychotherapy, most notably CBT-T, has attracted particular interest in recent years. As an innovative approach, CBT-T circumvents major obstacles to effective treatment of latelife anxiety disorders (e.g., limited access due to physical impairment or given that agoraphobia, as the most frequent anxiety disorder in late life, prevents patients from leaving their home [50]). Moreover, the home-based setting ensures privacy, which accommodates the needs of older adults with an anxiety disorder. Several studies have demonstrated the effectiveness of CBT-T in late-life anxiety disorders; a randomized controlled trial in 141 adults aged $\geq 60$ years with GAD reported a significantly greater decline in worry severity and GAD symptoms as assessed by HARS in the CBT-T group when compared to nondirective supportive therapy at the 4 month follow-up.
Comparable long-term effects of CBT-T were evident at the 15 month follow-up [51]. A secondary data analysis confirmed the superiority of CBT-T with a sustainable reduction of insomnia 1 year after completion of the treatment and improvement of the health-related quality of life [52]. Similarly, internet-based CBT has been proven effective in GAD producing moderate to large effect size reductions in symptom severity, distress, and impairment independently of age. Among the age group $>60$ years, a sustainable reduction in GAD 7 scores was evident [53].

\section{Prevention}

In view of the clear public health significance of latelife anxiety disorders, there is an urgent need to develop effective strategies for disease prevention. A series of studies proved the effectiveness of a stepped care prevention program combining a watchful-waiting approach, CBT-based bibliotherapy, CBT-based problem-solving treatment, and referral to primary care for medication. Implementation of the stepped care model in participants aged $\geq 75$ years with subthreshold of anxiety or depression yielded a significant reduction in the 12 -month incidence of anxiety and depressive disorders, with sustainable effects at the 24-month follow-up [54]. Effective prevention of late-life anxiety disorders will not only lower the individual and socioeconomic disease burden per se but also reduce sequential comorbidity with depression, sleep-related disorders, or substance use disorders. With regard to potentially beneficial effects of preventive measures in late life on cognitive decline or dementia, detection of elevated anxiety symptoms in combination with high-risk fluid and PET biomarkers of dementia such as $\mathrm{A} \beta$ deposition, pathologic $\tau$, and neurodegeneration may help to identify participants for future targeted prevention trials.

\section{Conclusion}

Taken together, heightened awareness and a more accurate diagnosis as well as deeper knowledge about the biological and biographical underpinnings of latelife anxiety disorders conferring an as yet underestimated individual and societal burden are required for informed decisions on more effective treatment including personalized approaches, inclusion into therapy trials (e.g., enrichment of patient populations for novel therapies), and preventive strategies in primary and home-based care as well as in specialized facilities. In 
light of the female-dominant prevalence of anxiety disorders and female-specific risk factors, consideration of developmental stages and hormonal status as well as sex-specific differences in clinical presentation is essential for clinical assessment and treatment recommendations.

\section{Acknowledgement}

We would like to thank Dr. Simon Maier for his assistance with interpretation of the fMRI data.

\section{Statement of Ethics}

The authors have no ethical conflicts to disclose.

\section{Disclosures Statement}

The authors have no conflict of interests to declare.

\section{Funding Sources}

This work was supported by the Deutsche Forschungsgemeinschaft (DFG; German Research Foundation) - project No. 44541416 - TRR 58 "Fear, Anxiety, Anxiety Disorders," within projects $\mathrm{C} 02$ and Z02 (to K.D.).

\section{Author Contributions}

S.H. and K.D. performed the literature search, evaluated and selected papers to include in this review, wrote this paper, and approved the final version of this work.

\section{References}

1 Wolitzky-Taylor KB, Castriotta N, Lenze EJ, Stanley MA, Craske MG. Anxiety disorders in older adults: a comprehensive review. Depress Anxiety. 2010 Feb;27(2):190-211.

2 Brenes GA, Miller ME, Stanley MA, Williamson JD, Knudson M, McCall WV. Insomnia in older adults with generalized anxiety disorder. Am J Geriatr Psychiatry. 2009 Jun;17(6): $465-72$.

3 Voyer P, Preville M, Roussel ME, Berbiche D, Beland SG. Factors associated with benzodiazepine dependence among communitydwelling seniors. J Community Health Nurs. 2009 Jul;26(3):101-13.

4 Gimson A, Schlosser M, Huntley JD, Marchant NL. Support for midlife anxiety diagnosis as an independent risk factor for dementia: a systematic review. BMJ Open. 2018 Apr; 8(4):e019399.

5 Becker E, Orellana Rios CL, Lahmann C, Rücker G, Bauer J, Boeker M. Anxiety as a risk factor of Alzheimer's disease and vascular dementia. Br J Psychiatry. 2018 Nov;213(5): 654-60.

6 Hanseeuw BJ, Jonas V, Jackson J, Betensky RA, Rentz DM, Johnson KA, et al. Association of anxiety with subcortical amyloidosis in cognitively normal older adults. Mol Psychiatry. DOI: 10.1038/s41380-018-0214-2.

7 Tawakol A, Ishai A, Takx RA, Figueroa AL, Ali A, Kaiser Y, et al. Relation between resting amygdalar activity and cardiovascular events: a longitudinal and cohort study. Lancet. 2017 Feb;389(10071):834-45.

8 Hernandez R, Allen NB, Liu K, Stamler J, Reid $\mathrm{KJ}$, Zee PC, et al. Association of depressive symptoms, trait anxiety, and perceived stress with subclinical atherosclerosis: results from the Chicago Healthy Aging Study (CHAS). Prev Med. 2014 Apr;61:54-60.
9 Holwerda TJ, Schoevers RA, Dekker J, Deeg DJ, Jonker C, Beekman AT. The relationship between generalized anxiety disorder, depression and mortality in old age. Int J Geriatr Psychiatry. 2007 Mar;22(3):241-9.

10 Laan W, Termorshuizen F, Smeets HM, Boks MP, de Wit NJ, Geerlings MI. A comorbid anxiety disorder does not result in an excess risk of death among patients with a depressive disorder. J Affect Disord. 2011 Dec;135(1-3): 284-91.

11 Batty GD, Russ TC, Stamatakis E, Kivimaki M. Psychological distress in relation to site specific cancer mortality: pooling of unpublished data from 16 prospective cohort studies. BMJ 2017;356:j108.

12 Gottschalk MG, Domschke K. Novel developments in genetic and epigenetic mechanisms of anxiety. Curr Opin Psychiatry. 2016 Jan; 29(1):32-8

13 Lee LO, Gatz M, Pedersen NL, Prescott CA. Anxiety trajectories in the second half of life: genetic and environmental contributions over age. Psychol Aging. 2016 Feb;31(1):10113.

14 Holmes SE, Esterlis I, Mazure CM, Lim YY, Ames D, Rainey-Smith S, et al. Australian Imaging, Biomarkers, Lifestyle Research Group: Beta-amyloid, apoe and bdnf genotype, and depressive and anxiety symptoms in cognitively normal older women and men. Am J Geriatr Psychiatry. 2016;24:1191-5.

15 Lenze EJ, Goate AM, Nowotny P, Dixon D, Shi P, Bies RR, et al. Relation of serotonin transporter genetic variation to efficacy of escitalopram for generalized anxiety disorder in older adults. J Clin Psychopharmacol. 2010 Dec;30(6):672-7.

16 Grünblatt E, Löffler C, Zehetmayer S, Jungwirth S, Tragl KH, Riederer P, et al. Associa- tion study of the 5-HTTLPR polymorphism and depression in 75-Year-Old nondemented subjects from the Vienna Transdanube Aging (VITA) study. J Clin Psychiatry. 2006 Sep; 67(9):1373-8.

17 Schiele MA, Domschke K. Epigenetics at the crossroads between genes, environment and resilience in anxiety disorders. Genes Brain Behav. 2018 Mar;17(3):e12423.

18 Andreescu C, Tudorascu D, Sheu LK, Rangarajan A, Butters MA, Walker S, et al. Brain structural changes in late-life generalized anxiety disorder. Psychiatry Res Neuroimaging. 2017 Oct;268:15-21.

19 Andreescu C, Aizenstein H. MRI studies in late-life mood disorders. Curr Top Behav Neurosci. 2012;11:269-87.

20 Andreescu C, Gross JJ, Lenze E, Edelman KD, Snyder S, Tanase C, et al. Altered cerebral blood flow patterns associated with pathologic worry in the elderly. Depress Anxiety. 2011 Mar;28(3):202-9.

21 Karim HT, Tudorascu DL, Butters MA, Walker S, Aizenstein HJ, Andreescu C. In the grip of worry: cerebral blood flow changes during worry induction and reappraisal in late-life generalized anxiety disorder. Transl Psychiatry. 2017 Aug;7(8):e1204.

22 Andreescu C, Sheu LK, Tudorascu D, Gross JJ, Walker S, Banihashemi L, et al. Emotion reactivity and regulation in late-life generalized anxiety disorder: functional connectivity at baseline and post-treatment. Am J Geriatr Psychiatry. 2015 Feb;23(2):200-14.

23 Andreescu C, Sheu LK, Tudorascu D, Walker $\mathrm{S}$, Aizenstein $\mathrm{H}$. The ages of anxiety-differences across the lifespan in the default mode network functional connectivity in generalized anxiety disorder. Int J Geriatr Psychiatry. 2014 Jul;29(7):704-12. 
24 Beck AT, Clark DA. An information processing model of anxiety: automatic and strategic processes. Behav Res Ther. 1997 Jan;35(1): 49-58.

25 Price RB, Eldreth DA, Mohlman J. Deficient prefrontal attentional control in late-life generalized anxiety disorder: an fMRI investigation. Transl Psychiatry. 2011 Oct;1(10):e46.

26 Mantella RC, Butters MA, Amico JA, Mazumdar S, Rollman BL, Begley AE, et al. Salivary cortisol is associated with diagnosis and severity of late-life generalized anxiety disorder. Psychoneuroendocrinology. 2008 Jul; 33(6):773-81.

27 Lenze EJ, Dixon D, Mantella RC, Dore PM, Andreescu C, Reynolds CF 3rd, et al. Treatment-related alteration of cortisol predicts change in neuropsychological function during acute treatment of late-life anxiety disorder. Int J Geriatr Psychiatry. 2012 May;27(5): 454-62.

28 Rosnick CB, Wetherell JL, White KS, Andreescu C, Dixon D, Lenze EJ. Cognitive-behavioral therapy augmentation of SSRI reduces cortisol levels in older adults with generalized anxiety disorder: A randomized clinical trial. J Consult Clin Psychol. 2016 Apr;84(4): 345-52.

29 Cover KK, Maeng LY, Lebrón-Milad K, Milad MR. Mechanisms of estradiol in fear circuitry: implications for sex differences in psychopathology. Transl Psychiatry. 2014 Aug; 4(8):e422.

30 Bromberger JT, Kravitz HM, Chang Y, Randolph JF Jr, Avis NE, Gold EB, et al. Does risk for anxiety increase during the menopausal transition? Study of women's health across the nation. Menopause. 2013 May;20(5):48895.

31 Claudia P, Andrea C, Chiara C, Stefano L, Giuseppe M, Vincenzo DL, et al. Panic disorder in menopause: a case control study. Maturitas. 2004 Jun;48(2): 147-54.

32 Gleason CE, Dowling NM, Wharton W, Manson JE, Miller VM, Atwood CS, et al. Effects of hormone therapy on cognition and mood in recently postmenopausal women: findings from the randomized, controlled keeps-cognitive and affective study. PLoS Med. 2015 Jun;12(6):e1001833.

33 Scali J, Ryan J, Carrière I, Ritchie K, Ancelin ML. A prospective study of hormonal treatment and anxiety disorders in community-dwelling elderly women (the Esprit Study). J Affect Disord. 2009 May;115(1-2): 274-9.
34 Hantsoo L, Epperson CN. Anxiety disorders among women: A female lifespan approach. Focus Am Psychiatr Publ. 2017;15(2):162-72.

35 Chou KL, Mackenzie CS, Liang K, Sareen J. Three-year incidence and predictors of firstonset of DSM-IV mood, anxiety, and substance use disorders in older adults: results from Wave 2 of the National Epidemiologic Survey on Alcohol and Related Conditions. J Clin Psychiatry. 2011 Feb;72(2):144-55.

36 Lyketsos CG, Lopez O, Jones B, Fitzpatrick AL, Breitner J, DeKosky S. Prevalence of neuropsychiatric symptoms in dementia and mild cognitive impairment: results from the cardiovascular health study. JAMA. 2002 Sep; 288(12):1475-83

37 Schultz SK, Moser DJ, Bishop JR, Ellingrod VL. Phobic anxiety in late-life in relationship to cognition and 5HTTLPR polymorphism. Psychiatr Genet. 2005 Dec;15(4):305-6.

38 Wilkes CM, Wilson HW, Woodard JL, Calamari JE. Do negative affect characteristics and subjective memory concerns increase risk for late life anxiety? J Anxiety Disord. 2013 Aug; 27(6):608-18.

39 Mohlman J, Gorman JM. The role of executive functioning in CBT: a pilot study with anxious older adults. Behav Res Ther. 2005 Apr;43(4):447-65.

40 Steiner AR, Petkus AJ, Nguyen H, Wetherell JL. Information processing bias and pharmacotherapy outcome in older adults with generalized anxiety disorder. J Anxiety Disord. 2013 Aug;27(6):592-7.

41 Ong AD, Uchino BN, Wethington E. Loneliness and health in older adults: A mini-review and synthesis. Gerontology. 2016;62(4):4439.

42 van Beljouw IM, Verhaak PF, Cuijpers P, van Marwijk HW, Penninx BW. The course of untreated anxiety and depression, and determinants of poor one-year outcome: a one-year cohort study. BMC Psychiatry. 2010 Oct; 10(1):86.

43 Flensborg-Madsen T, Tolstrup J, Sørensen HJ, Mortensen EL. Social and psychological predictors of onset of anxiety disorders: results from a large prospective cohort study. Soc Psychiatry Psychiatr Epidemiol. 2012 May;47(5):711-21.

44 Mohlman J, Bryant C, Lenze EJ, Stanley MA, Gum A, Flint A, et al. Improving recognition of late life anxiety disorders in Diagnostic and Statistical Manual of Mental Disorders, Fifth Edition: observations and recommendations of the advisory committee to the lifespan dis- orders work group. Int J Geriatr Psychiatry 2012;27:549-56.

45 Pachana NA, Byrne GJ, Siddle H, Koloski N, Harley E, Arnold E. Development and validation of the geriatric anxiety inventory. Int Psychogeriatr. 2007 Feb;19(1):103-14.

46 Segal DL, June A, Payne M, Coolidge FL, Yochim B. Development and initial validation of a self-report assessment tool for anxiety among older adults: the Geriatric Anxiety Scale. J Anxiety Disord. 2010 Oct;24(7):709-14.

47 Gerolimatos LA, Gregg JJ, Edelstein BA. Assessment of anxiety in long-term care: examination of the Geriatric Anxiety Inventory (GAI) and its short form. Int Psychogeriatr. 2013 Sep;25(9):1533-42.

48 Lueken U, Zierhut KC, Hahn T, Straube B, Kircher T, Reif A, et al. Neurobiological markers predicting treatment response in anxiety disorders: a systematic review and implications for clinical application. Neurosci Biobehav Rev. 2016 Jul;66:143-62.

49 Lenze EJ, Hershey T, Newcomer JW, Karp JF, Blumberger D, Anger J, et al. Antiglucocorticoid therapy for older adults with anxiety and co-occurring cognitive dysfunction: results from a pilot study with mifepristone. Int J Geriatr Psychiatry. 2014 Sep;29(9):962-9.

50 Canuto A, Weber K, Baertschi M, Andreas S, Volkert J, Dehoust MC, et al. Anxiety disorders in old age: psychiatric comorbidities, quality of life, and prevalence according to age, gender, and country. Am J Geriatr Psychiatry. 2018 Feb;26(2):174-85.

51 Brenes GA, Danhauer SC, Lyles MF, Anderson A, Miller ME. Long-term effects of telephone-delivered psychotherapy for late-life gad. Am J Geriatr Psychiatry. 2017 Nov; 25(11):1249-57.

52 Brenes GA, Danhauer SC, Lyles MF, Anderson A, Miller ME. Effects of telephone-delivered cognitive-behavioral therapy and nondirective supportive therapy on sleep, healthrelated quality of life, and disability. Am Geriatr Psychiatry. 2016 Oct;24(10):846-54.

53 Hobbs MJ, Mahoney AE, Andrews G. Integrating $\mathrm{iCBT}$ for generalized anxiety disorder into routine clinical care: treatment effects across the adult lifespan. J Anxiety Disord. 2017 Oct;51:47-54.

54 van't Veer-Tazelaar PJ, van Marwijk HW, van Oppen P, van der Horst HE, Smit F, Cuijpers $\mathrm{P}$, et al. Prevention of late-life anxiety and depression has sustained effects over 24 months: a pragmatic randomized trial. Am J Geriatr Psychiatry. 2011 Mar;19(3):230-9. 